\title{
Rapport annuel 2015
}

Au cours de l'exercice écoulé, la Caisse de secours des médecins suisses a accordé son soutien à 15 médecins tombés malgré eux dans le besoin (ils étaient 11 en 2014) ainsi qu'aux proches et aux descendants de médecins décédés.

Trois aides durables et une aide unique se sont ajoutées en 2015.

Les produits ont résulté des recettes provenant de dons pour un montant de CHF 114405.90 (2014: 108006) ainsi que des intérêts et des dividendes du capital de la fondation, à hauteur de CHF 86633.30 (2014: 69407).

Concernant les charges, les contributions de soutien que nous avons versées ont atteint le montant de CHF 132000 (2014: 95000). Les dépenses relatives à la gestion de la Caisse de secours et à l'administration de la fortune se sont élevées à CHF 68800 .

La différence entre les recettes provenant de dons ainsi que des intérêts et dividendes du capital et les charges consécutives aux contributions de soutien et aux dépenses administratives a constitué un surplus de recettes de CHF 308.65 affecté à la fortune de la fondation.

Les dépenses de gestion et d'administration de la fortune peuvent sembler très élevées, mais elles sont nécessaires pour que la fondation soit solide et conforme aux directives - sans cela, nous ne pourrions pas satisfaire aux examens exigés d'office d'une fondation bénéficiant d'une exonération fiscale totale. Cette dernière nous permet de recevoir de temps en temps un don d'un montant plus élevé.

Les dons individuels inférieurs à CHF 500 forment le pilier traditionnel de notre fondation. Afin de réduire nos frais administratifs, nous renonçons à remercier personnellement les donateurs. Nous leur exprimons donc notre gratitude ici.
Les comptes annuels de la Caisse de secours ont été révisés par la société Marin Treuhand AG qui en a confirmé l'exactitude. Nous la remercions cordialement de son travail. Des informations détaillées sur les comptes annuels sont disponibles dans le bilan et le compte d'exploitation publiés ci-après.

La fortune de la fondation consiste en un mélange d'obligations et d'actions satisfaisant aux prescriptions de la prévoyance professionnelle (LPP).

Le compte d'exploitation, le bilan et la gestion de la fortune sont examinés chaque année par l'autorité de surveillance LPP et des fondations des deux Bâle. L'activité du Conseil de fondation est bénévole.

Je tiens à remercier tous les membres de la Commission pour le travail fourni l'an passé. Je remercie également Messieurs J. Kobler et A. Billich de Notenstein La Roche Banque Privée SA pour la tenue de la comptabilité et la gestion de la fortune.

La Commission de fondation de la Caisse de secours des médecins suisses

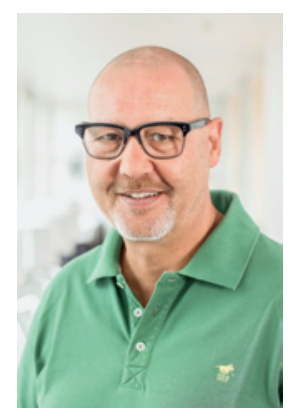

Dr U. Lüscher, président (photo)

DrS. Stöhr

Dr U. Leibundgut, trésorier

Compte postal de la Caisse de secours des médecins suisses: 40-644-3 Bâle 


\section{Comptes annuels 2015}

\begin{tabular}{|c|c|c|c|}
\hline Bilan au 31 Décembre 2015 & & Actifs & Passifs \\
\hline & & $\mathrm{CHF}$ & $\mathrm{CHF}$ \\
\hline Compte postal & & 181950.82 & \\
\hline Débiteur impôt anticipé & & 21152.90 & \\
\hline Comptes de régularisation des actifs & & 42207.19 & \\
\hline Compte de régularisation des passifs & & & 4160.00 \\
\hline \multicolumn{4}{|l|}{ Fortune au 31 Décembre 2014} \\
\hline Caisse de secours au 01.01. & & & 3280727.98 \\
\hline
\end{tabular}

\begin{tabular}{|c|c|c|}
\hline \multirow[t]{2}{*}{ Compte d'exploitation pour 2015} & Charges & Produits \\
\hline & $\mathrm{CHF}$ & $\mathrm{CHF}$ \\
\hline Revenu sur capital & & 86633.30 \\
\hline Ajustement provision sur titres & & 15000.00 \\
\hline Contributions & 132000.00 & \\
\hline Frais de banque & 28825.32 & \\
\hline Frais d'administration & 39974.72 & \\
\hline Bénéfice de l'exercice & 308.65 & \\
\hline
\end{tabular}

